SESSION E

PROPER MOTIONS AND GALACTIC PROBLEMS 


\title{
REMARKS ON THE DEFINITION AND DETERMINATION OF PROPER MOTIONS
}

\author{
(Invited Paper)
}

\author{
W. DIECK VOSS
}

Hamburger Sternwarte, F.R. Germany

\begin{abstract}
Classical methods of compiling catalogues of proper motions from catalogues of positions at different epochs and reduction to a rigorously defined homogeneous coordinate-system are reviewed, and the determination of proper motions of numerous stars by photographic differential methods, partly with assumptions on behaviour of faint reference stars, and partly by attachment to reference frames of stars with proper motions known from catalogues is discussed.
\end{abstract}

1. The proper motion of a star is defined as the time rate of change of its position on the celestial sphere. The proper motion corresponds to the apparent transverse velocity in a fixed coordinate system, or a projection of the true velocity onto the tangential plane. The effect of the usual procedure is that the time variable is expressed in ephemeris time, because the epochs given for positions are calculated from the beginning of the relevant Besselian year, which in turn is linked with the motion of the Earth around the Sun. Except for Barnard's star, Groombridge 1830, and perhaps one or two other stars, the motion of a star may always be represented by a uniform motion in a great circle. Strictly speaking this applies to the centre of gravity in the case of a multiple system. It is only for very nearby stars with appreciable radial velocity that the second order time derivative of position enters, which gives the opportunity of checking astrometric data.

By representing the proper motion as apparent velocity in a great circle, say, in seconds of arc per century (with current precision, it is not necessary to distinguish between Ephemeris, Tropical or Julian centuries), and by specifying the position angle of the motion in a fixed equatorial system, it is possible to avoid singularities near the celestial poles. The two positions $\alpha_{1}, \delta_{1} ; \alpha_{2}, \delta_{2}$ for the two epochs $t_{1}, t_{2}$ are interconnected by the following formulae, where $\Delta \alpha=\alpha_{2}-\alpha_{1}$, the displacement is expressed by $w$, and the position-angle by $P$ :

$$
\begin{aligned}
& \sin w \sin P=\cos \delta_{2} \sin \Delta \alpha \\
& \sin w \cos P=\cos \delta_{1} \sin \delta_{2}-\sin \delta_{1} \cos \delta_{2} \cos \Delta \alpha \\
& \cos w \quad=\sin \delta_{1} \sin \delta_{2}+\cos \delta_{1} \cos \delta_{2} \cos \Delta \alpha
\end{aligned}
$$

and

$$
\begin{aligned}
\cos \delta_{2} \sin \Delta \alpha & =\sin P \sin w \\
\cos \delta_{2} \cos \Delta \alpha & =\cos \delta_{1} \cos w-\sin \delta_{1} \sin w \cos P \\
\sin \delta_{2} & =\sin \delta_{1} \cos w+\cos \delta_{1} \sin w \cos P
\end{aligned}
$$


When $w$ is small in (1) one may substitute

$$
\cos \Delta \alpha \sim 1, \quad \sin \Delta \alpha \sim \Delta \alpha, \quad \sin w \sim w
$$

and one gets the usual approximation in the tangential plane:

$$
\begin{aligned}
& w \sin P=\Delta \alpha \cos \delta_{2} \\
& w \cos P=\delta_{2}-\delta_{1}
\end{aligned}
$$

If $\mu$ stands for the total proper motion, then $w=\mu\left(t_{2}-t_{1}\right)$.

2. The procedure of deriving proper motion from positions at different epochs seems to be straightforward. In detail there are several complications involved. The most important will be the necessity of creating a common coordinate system conforming to an established fundamental system of positions and proper motions. At this point the real difficulties show up. Change on account of precession in the case of different equinoxes of the two catalogues is simple as long as there is a consensus on the precessional constants. But it often becomes necessary to take into account changes of constants between the time when the two catalogues were being prepared for publication. Thus if somebody makes excerpts from the Geschichte des Fixsternhimmels he has to bear in mind that here the reductions to the normal equinox of 1875.0 were performed with Struve's constant of precession while later on Newcomb's constant was generally used. The reduction to a unified system including the realisation of a true fundamental system is a real difficulty requiring ingenuity and experience.

The wealth of information on the astrometrically more important stars is gradually increasing owing to the perseverance of observers who prepare new catalogues of position; the biggest example here is the work of Yale Observatory. In most cases not only positions but also values of proper motion are published. In the routine work positions from older catalogues are introduced and the proper motions are printed in columns adjacent to the positions. With this kind of presentation the positions may be updated to any chosen epoch, but it is inadequate for improvement by the addition of further observations.

In the international undertaking AGK3, originally planned for merely furnishing (quickly) proper motions for all the stars of the AGK2, the AGK2 was revised by means of the original rectangular coordinates and, after applying systematic corrections, a new edition was produced in the fundamental system of the FK4. The magnetic tapes and the manuscript (in preparation) give positions at both epochs; the printed proper motions were derived by taking the differences of positions and dividing these by the differences in epoch. The proper motions in right ascension are multiplied by the cosine of the declination in AGK3; the differences in epoch are also given. Thus it is possible not only to update the positions to other epochs but to determine the mean errors for these epochs, and to add new observations easily. In the case of more than two observations, application of Newcomb's method 
of the central date is appropriate. The addition of new observations was a special concern of the author of the General Catalogue (B. Boss, 1937, Vol. 1, p. 49).

In principle the zero of epoch might be chosen arbitrarily while fitting a great circle through the positions at different epochs and with different weights by least squares. If the zero of epoch is taken as the weighted mean of the epochs (the "central date'), the resulting position for this zero epoch has maximum weight and is not dependent on the proper motions. Therefore in this case the mean error for any other epoch may be calculated directly by means of the law of propagation of errors. Furthermore, addition of new observations is possible without bias. In the present age of computers one is no longer compelled to separate the two coordinates right ascension and declination, and the fitting of observations by a great circle motion and a position angle seems to be easy enough.

3. It has often been argued that it is dangerous to use large collections of proper motions of moderate precision for the study of the kinematical behaviour of the stars in the Sun's neighbourhood. Indeed we are no longer satisfied just to repeat the pioneer work of deriving secular parallaxes from proper motions of stars of different apparent magnitude and of different galactic latitude. Nevertheless, we shall need to use the tables derived from such work for some time to come, and in the absence of other information, the reduction of plates with mean proper motion of faint stars will continue. There is a special danger in using proper motions of moderate precision for statistical purposes. The distances of the individual stars are unknown, and the restriction to directions of the proper motion recommends itself. But even here in the majority of cases the proper motion is rather small and the direction becomes meaningless, and what one is doing in averaging proper motions in small regions of the sky remains rather obscure.

In this context I would like to recall the simple concept of reduced proper motion. Assuming the absolute magnitudes of a selected class of stars to be constant, a rectification to a uniform unit of tangential velocity is possibly by reducing the proper motion $\mu$ of a star of magnitude $m$ to a standard magnitude $m_{0}$ by

$$
\log \mu^{\prime}=\log \mu+0.2\left(m-m_{0}\right) \text {. }
$$

Thus the concept of a mean proper motion of a selected class of stars, supposed to be within a restricted range of distances in a selected region in the sky, may be transformed to the average behaviour of a whole cloud of stars surrounding the Sun, and the selected region, by inclusion of stars in a large range of apparent magnitudes, may be replaced by a selected plane through the Sun. The reduced proper motion then may be visualized as the tangential components of the space velocities; further reduction into units of $\mathrm{km} \mathrm{s}^{-1}$ or $\mathrm{AU} \mathrm{\textrm {yr } ^ { - 1 }}$ is then possible by using a common factor.

At the computing centre in Hamburg, I have the AGK3 reduced to galactic coordinates, in background storage. The standard values of Oort's rotation constants and the values recommended by Fricke for correction of precessional constants have been applied. In due course with a proper weighting system, to diminish the influence 
of proper motions which are small compared with their mean errors, I hope to investigate the solar apex and axes of the velocity ellipsoid for various spectral classes.

I perhaps may be permitted to offer a little warning; with the invention and installation of modern astrographs and with scanning and measuring machines operating automatically, it becomes possible to handle millions of positions and proper motions using methods of reduction which promise excellent returns in relation to effort. Before engaging in a large programme, considerable thought must be given to its objective and to what additional data such as magnitudes, colours and spectral types, may be necessary. Some positional catalogues already contain such data.

4. Special objects for proper motion study may be anything astronomers are interested in; such as classes of variable stars which may be used for studying the distance scale of the Universe, calibration of luminosity classes, moving clusters, and even globular clusters. The latter, on account of their large distances, are well suited for studying the behaviour of the surrounding field stars. The brighter stars normally have a history from which proper motions can be determined by the methods which I have already described. With powerful instruments of longer focal length, photographic methods come into the picture. Pairs of plates taken with the same instrument at different epochs give the coordinate differences in units of - say - $\mathrm{mm}$. Using least squares, with linear formulae in the coordinates, the relative scale and orientation of the plates can be determined from reference stars near the edge of the field. The residuals are then converted into relative proper motion by applying a mean scale value ("/mm) and the time interval. The relative proper motions can then be reduced to an absolute system by assuming the average motion of a number of faint reference stars. Alternatively, if stars with known fundamental proper motions are included in the measures, the zero-point of proper motions may be referred to the fundamental system, provided that no serious magnitude error is thereby introduced. It should be stressed that, in any case, the scale value must be known a priori, and not derived from the adopted proper motions.

In cluster work it is a good policy to use the member stars themselves as a reference frame in order to achieve a good plate solution. Reference stars in the field may once more serve to reduce the proper motions to the fundamental system.

In conclusion I would like to stress the general importance of proper motion work in Kapteyn's selected areas; here we have excellent samples of data that are particularly suitable for statistical studies into greater depths of the galactic system.

\section{DISCUSSION}

Luyten: If it is appropriate to mention it here - since Dr Dieckvoss has spoken of proper motions for special objects, I should like to mention that since I have both the original Palomar Survey plates as well as repeats of all the red plates, I am in a position to determine the proper motion for any object brighter than $21 m_{p g}$ north of $-33^{\circ}(1975)$.

Eichhorn: Rigorous formulae have recently been published in the Astronomische Nachrichten which give right ascensions and declinations at any epoch as rigorous functions of initial position, proper motion, 
and ratio of radial velocity to distance, as well as the proper motion components and the radial velocity over distance ratio at any epoch as functions of the above-mentioned values at a zero epoch. It should be pointed out that the second order formulae in most, if not all, text books are wrong since they take no account of the radial velocity to distance ratio. Since completely rigorous formulae are now available, I believe that they should be used, lest investigators come to regard the incomplete second order formulae as completely satisfactory for all stars and all epoch differences.

Dieckvoss: At present time this only applies to two stars, Barnard's, and Groombridge 1830.

Bok: Secular parallaxes for stars at large are of little use - BUT - secular parallaxes for special groups, $\mathrm{K}$ giants or dwarfs, for example can be very useful.

Proper motions are urgently needed for RR Lyrae variables. Dr van Herk has work under way for 400 of these stars and this project - combined with the McCormick work - should be very important for calibration purposes of absolute magnitudes. Radial velocity work (with image tubes!) on RR Lyrae stars with known p.m.'s promises to yield important results relating to the scale of our Galaxy and related problems.

Fricke: My comment consists of two parts, the first concerning Dr Eichhorn's remark on the variation of proper motions due to the foreshortening effect. This effect is very small, and the formulae given in the textbooks have been sufficient for most of the practical purposes. I think that people who derive highly accurate proper motions will not use textbooks but more rigorous formulae whenever this is necessary. Second, I want to comment on the effects of inaccurate proper motions on kinematical results. For stars nearer than about $100 \mathrm{pc}$ from the Sun the effects of precessional corrections and systematic differences between modern catalogues are small in the transverse motions to be derived. Their importance grows with increasing distance from the Sun, and they all have to be taken into account in kinematics of stars farther away than $100 \mathrm{pc}$. For distances larger than about $1500 \mathrm{pc}$, even the best available proper motions (with m.e. of \pm 0 "'20 per century) fail to contribute to significant results. Furthermore, I want to say that the results of a good number of older determinations of secular parallaxes may be wrong due to the fact that the material on which they were based does not fulfil the conditions which underly the equations of determination.

Bok: Dr Fricke's condemnation of secular parallaxes is obviously justified for research at low galactic latitudes. I take the view that secular parallaxes at high and at intermediate galactic latitudes can serve a very useful purpose in checking on any conclusions relating to density and velocity distributions of special groups of stars perpendicular to the galactic plane. Secular parallaxes should be most useful in providing us with checks on the tilts of equidensity surfaces at various points above and below.

Fricke: I should have certainly specified in more detail my criticism of secular parallax determinations. One needs to know photometric data and MK spectral classes (except that one is dealing with a class of variables) in order to arrive at a significant luminosity determination. Furthermore, it is not possible to average over a large distance interval when the distribution of stars is not Gaussian about a 'mean' distance, or when the distribution deviates appreciably from a uniform one. 